\title{
Nonlinear Super Integrable Couplings of Super C-KdV Hierarchy
}

\author{
Si-Xing Tao
}

\begin{abstract}
Enlargement of Lie super algebra $\mathrm{B}(0,1)$ was given firstly. Then nonlinear super integrable couplings of the super C-KdV hierarchy based upon this enlarged matrix Lie super algebra were constructed secondly. And its super Hamiltonian structures were established by using super trace identity thirdly. As its reduction, special integrable couplings of the C-KdV hierarchy were obtained finally.
\end{abstract}

Index Terms - Lie super algebra, super integrable couplings, super C-KdV hierarchy, super Hamiltonian structures.

\section{INTRODUCTION}

With the development of soliton theory, super integrable systems associated with Lie super algebra have aroused growing attentions by many mathematicians and physicists. It was known that super integrable systems contained the odd variables, which would provide more prolific fields for mathematical researchers and physical ones. Several super integrable systems including super AKNS hierarchy, super KdV hierarchy, super C-KdV hierarchy and super classical Boussinseq hierarchy, etc., have been studied[1]-[4]. There are some interesting results on the super integrable systems, such as Darboux transformation [5], super Hamiltonian structures[6], [7], binary nonlinearization[8] and reciprocal transformation[9] and so on.

The research of integrable couplings of the well known integrable hierarchy has been received considerable attentions[10]-[15]. A few approaches to construct linear integrable couplings of the classical soliton equation are presented by permutation, enlarging spectral problem, using matrix Lie algebra constructing new loop Lie algebra [16] and creating semi-direct sums of Lie algebra. Recently, Ma and Zhu [17], [18] presented a scheme for constructing nonlinear continuous and discrete integrable couplings using the block type matrix algebra. However, there is one interesting question for us is how to generate nonlinear super integrable couplings for the super integrable hierarchy.

In this paper, we hope to construct nonlinear super integrable couplings of the super soliton equations through enlarging matrix Lie super algebra. We take the Lie algebra $B(0,1)$ as an example to illustrate the approach for extending

Manuscript received April 8, 2013; revised June 28, 2103. This work was supported by the National Natural Science Foundation of China under Grant No.61072147, the Natural Science Foundation of Henan Province under Grant No.132300410202, the Science and Technology Key Research Foundation of the Education Department of Henan Province under Grant No.12A110017 and the Youth Research Foundation of Shangqiu Normal University under Grant No. 2011QN12.

S. X. Tao is with School of Mathematics and Information Science, Shangqiu Normal University, Henan, Shangqiu 476000, China (e-mail: taosixing@163.com).
Lie super algebras. Based on the enlarged Lie super algebra $g l(6,2)$, we work out nonlinear super integrable Hamiltonian couplings of the super C-KdV hierarchy. Finally, we will reduce the nonlinear super $\mathrm{C}-\mathrm{KdV}$ integrable Hamiltonian couplings to some special cases.

\section{ENLARGEMENT OF LIE SUPER AlgEBRA}

Consider the Lie super algebra $B(0,1)$. Its basis is

$$
\begin{aligned}
& E_{1}=\left(\begin{array}{ccc}
1 & 0 & 0 \\
0 & -1 & 0 \\
0 & 0 & 0
\end{array}\right), E_{2}=\left(\begin{array}{lll}
0 & 1 & 0 \\
0 & 0 & 0 \\
0 & 0 & 0
\end{array}\right), E_{3}=\left(\begin{array}{lll}
0 & 0 & 0 \\
1 & 0 & 0 \\
0 & 0 & 0
\end{array}\right), \\
& E_{4}=\left(\begin{array}{ccc}
0 & 0 & 1 \\
0 & 0 & 0 \\
0 & -1 & 0
\end{array}\right), E_{5}=\left(\begin{array}{lll}
0 & 0 & 0 \\
0 & 0 & 1 \\
1 & 0 & 0
\end{array}\right) .
\end{aligned}
$$

where $E_{1}, E_{2}, E_{3}$ are even element and $E_{4}, E_{5}$ are odd elements. Their non-zero (anti) commutation relations are

$$
\begin{aligned}
& {\left[E_{1}, E_{2}\right]=2 E_{2},\left[E_{1}, E_{3}\right]=-2 E_{3},\left[E_{1}, E_{4}\right]=E_{4},} \\
& {\left[E_{1}, E_{5}\right]=-E_{5},\left[E_{2}, E_{3}\right]=E_{1},\left[E_{2}, E_{5}\right]=E_{4},} \\
& {\left[E_{3}, E_{4}\right]=E_{5},\left[E_{4}, E_{4}\right]=-2 E_{2},\left[E_{4}, E_{5}\right]=E_{1},} \\
& {\left[E_{5}, E_{5}\right]=2 E_{3} .}
\end{aligned}
$$

Let us enlarge the Lie super algebra $B(0,1)$ to the Lie super algebra $g l(6,2)$ with a basis

$$
\begin{aligned}
& e_{1}=\left(\begin{array}{ccccc}
1 & 0 & 0 & 0 & 0 \\
0 & -1 & 0 & 0 & 0 \\
0 & 0 & 1 & 0 & 0 \\
0 & 0 & 0 & -1 & 0 \\
0 & 0 & 0 & 0 & 0
\end{array}\right), e_{2}=\left(\begin{array}{ccccc}
0 & 1 & 0 & 0 & 0 \\
0 & 0 & 0 & 0 & 0 \\
0 & 0 & 0 & 1 & 0 \\
0 & 0 & 0 & 0 & 0 \\
0 & 0 & 0 & 0 & 0
\end{array}\right), \\
& e_{3}=\left(\begin{array}{lllll}
0 & 0 & 0 & 0 & 0 \\
1 & 0 & 0 & 0 & 0 \\
0 & 0 & 0 & 0 & 0 \\
0 & 0 & 1 & 0 & 0 \\
0 & 0 & 0 & 0 & 0
\end{array}\right), e_{4}=\left(\begin{array}{ccccc}
0 & 0 & 1 & 0 & 0 \\
0 & 0 & 0 & -1 & 0 \\
0 & 0 & 1 & 0 & 0 \\
0 & 0 & 0 & -1 & 0 \\
0 & 0 & 0 & 0 & 0
\end{array}\right), \\
& e_{5}=\left(\begin{array}{lllll}
0 & 0 & 0 & 1 & 0 \\
0 & 0 & 0 & 0 & 0 \\
0 & 0 & 0 & 1 & 0 \\
0 & 0 & 0 & 0 & 0 \\
0 & 0 & 0 & 0 & 0
\end{array}\right), e_{6}=\left(\begin{array}{lllll}
0 & 0 & 0 & 0 & 0 \\
0 & 0 & 1 & 0 & 0 \\
0 & 0 & 0 & 0 & 0 \\
0 & 0 & 1 & 0 & 0 \\
0 & 0 & 0 & 0 & 0
\end{array}\right), \\
& e_{7}=\left(\begin{array}{ccccc}
0 & 0 & 0 & 0 & 1 \\
0 & 0 & 0 & 0 & 0 \\
0 & 0 & 0 & 0 & 0 \\
0 & 0 & 0 & 0 & 0 \\
0 & -1 & 0 & 1 & 0
\end{array}\right), e_{8}=\left(\begin{array}{ccccc}
0 & 0 & 0 & 0 & 0 \\
0 & 0 & 0 & 0 & 1 \\
0 & 0 & 0 & 0 & 0 \\
0 & 0 & 0 & 0 & 0 \\
1 & 0 & -1 & 0 & 0
\end{array}\right) \text {. }
\end{aligned}
$$

where $e_{1}, e_{2}, e_{3}, e_{4}, e_{5}, e_{6}$ are even, and $e_{7}, e_{8}$ are odd.

The generator of Lie super algebra $g l(6,2), e_{i}(1 \leq i \leq 8)$ satisfy the following (anti) commutation relations: 
$\left[e_{1}, e_{2}\right]=2 e_{2},\left[e_{1}, e_{3}\right]=-2 e_{3},\left[e_{1}, e_{5}\right]=2 e_{5},\left[e_{1}, e_{6}\right]=-2 e_{6},\left[e_{1}, e_{7}\right]=e_{7}$,

$\left[e_{1}, e_{8}\right]=-e_{8},\left[e_{2}, e_{3}\right]=e_{1},\left[e_{2}, e_{4}\right]=-2 e_{5},\left[e_{2}, e_{6}\right]=e_{4},\left[e_{2}, e_{8}\right]=e_{7}$,

$\left[e_{3}, e_{4}\right]=2 e_{6},\left[e_{3}, e_{5}\right]=-e_{4},\left[e_{3}, e_{7}\right]=e_{8},\left[e_{4}, e_{5}\right]=2 e_{5},\left[e_{4}, e_{6}\right]=-2 e_{6}$,

$\left[e_{5}, e_{6}\right]=e_{4},\left[e_{7}, e_{7}\right]=2 e_{5}-2 e_{2},\left[e_{7}, e_{8}\right]=e_{1}-e_{4},\left[e_{8}, e_{8}\right]=2 e_{3}-2 e_{6}$,

$\left[e_{1}, e_{4}\right]=\left[e_{2}, e_{5}\right]=\left[e_{2}, e_{7}\right]=\left[e_{3}, e_{6}\right]=\left[e_{3}, e_{8}\right]=\left[e_{4}, e_{7}\right]=\left[e_{4}, e_{8}\right]=$

$\left[e_{5}, e_{7}\right]=\left[e_{5}, e_{8}\right]=\left[e_{6}, e_{7}\right]=\left[e_{6}, e_{8}\right]=0$.

Define a loop super algebra corresponding to the Lie super algebra $g l(6,2)$, denote by

$$
\begin{aligned}
\widetilde{g l}(6,2) & =g l(6,2) \otimes \mathbb{C}\left[\lambda, \lambda^{-1}\right] \\
& =\left\{e_{i} \lambda^{m} \mid e_{i} \in g l(6,2), i=1, \cdots, 8 ; m=0, \pm 1, \pm 2, \cdots\right\} .
\end{aligned}
$$

The corresponding (anti)commutative relations are given as

$$
\left[e_{i} \lambda^{m}, e_{j} \lambda^{n}\right]=\left[e_{i}, e_{j}\right] \lambda^{m+n}, \forall e_{i}, e_{j} \in g l(6,2)
$$

\section{NONLINEAR SUPER INTEGRABLE COUPLINGS OF SUPER C-KDV HIERARCHY}

Let us start from an enlarged spectral problem associated with $g l(6,2)$,

$$
\begin{aligned}
\phi_{x}= & U(u, \lambda) \phi, \\
U= & -\frac{1}{2} e_{1}(1)+\frac{1}{2} q e_{1}(0)-r e_{2}(0)+e_{3}(0) \\
& +u_{1} e_{4}(0)+u_{2} e_{5}(0)+\alpha e_{7}(0)+\beta e_{8}(0) \\
& \left(\begin{array}{ccccc}
-\frac{1}{2} \lambda+\frac{1}{2} q & -r & u_{1} & u_{2} & \alpha \\
1 & \frac{1}{2} \lambda-\frac{1}{2} q & 0 & -u_{1} & \beta \\
0 & 0 & -\frac{1}{2} \lambda+\frac{1}{2} q+u_{1} & -r+u_{2} & 0 \\
0 & 0 & 1 & \frac{1}{2} \lambda-\frac{1}{2} q-u_{1} & 0 \\
\beta & -\alpha & -\beta & \alpha & 0
\end{array}\right) .
\end{aligned}
$$

where $q, r, u_{1}, u_{2}$ are even potentials, but $\alpha, \beta$ are odd ones.

In order to obtain super integrable couplings of super integrable hierarchy, we first solve the adjoint representation of (2),

$$
V_{x}=[U, V]
$$

with

$$
\begin{aligned}
& G e_{6}(0)+\rho e_{7}(0)+\delta e_{8}(0) \\
= & \left(\begin{array}{ccccc}
A & B & E & F & \rho \\
C & -A & G & -E & \delta \\
0 & 0 & A+E & B+F & 0 \\
0 & 0 & C+G & -A-E & 0 \\
\delta & -\rho & -\delta & \rho & 0
\end{array}\right) .
\end{aligned}
$$$$
V=A e_{1}(0)+B e_{2}(0)+C e_{3}(0)+E e_{4}(0)+F e_{5}(0)+
$$

where $A, B, C, E, F$ and $G$ are commuting fields, and $\rho, \delta$ are anticommuting fields.

Substituting

$$
\begin{array}{r}
A=\sum_{m \geq 0} A_{m} \lambda^{-m}, B=\sum_{m \geq 0} B_{m} \lambda^{-m}, C=\sum_{m \geq 0} C_{m} \lambda^{-m}, E=\sum_{m \geq 0} E_{m} \lambda^{-m}, \\
F=\sum_{m \geq 0} F_{m} \lambda^{-m}, G=\sum_{m \geq 0} G_{m} \lambda^{-m}, \rho=\sum_{m \geq 0} \rho_{m} \lambda^{-m}, \delta=\sum_{m \geq 0} \delta_{m} \lambda^{-m} .(10)
\end{array}
$$

into above equation gives the following recursive formulas

$$
\left\{\begin{array}{l}
A_{m, x}=-B_{m}-r C_{m}+\beta \rho_{m}+\alpha \delta_{m}, \\
B_{m, x}=2 r A_{m}-B_{m+1}+\beta B_{m}-2 \alpha \rho_{m}, \\
C_{m, x}=2 A_{m}+C_{m+1}-q C_{m}+2 \beta \delta_{m}, \\
E_{m, x}=u_{2} C_{m}-F_{m}-r G_{m}+u_{2} G_{m}-\beta \rho_{m}-\alpha \delta_{m}, \\
F_{m, x}=-2 u_{2} A_{m}+2 u_{1} B_{m}+2 r E_{m}-2 u_{2} E_{m}-F_{m+1}+q F_{m}+2 u_{1} F_{m}+2 \alpha \rho_{m}, \\
G_{m, x}=-2 u_{1} C_{m}+2 E_{m}+G_{m+1}-q G_{m}-2 u_{1} G_{m}-2 \beta \delta_{m}, \\
\rho_{m, x}=-\alpha A_{m}-\beta B_{m}-\frac{1}{2} \rho_{m+1}+\frac{1}{2} q \rho_{m}-r \delta_{m}, \\
\delta_{m, x}=\beta A_{m}-\alpha C_{m}+\rho_{m}+\frac{1}{2} \delta_{m+1}-\frac{1}{2} q \delta_{m} .
\end{array}\right.
$$

From these equations, we can successively deduce $A_{0}=\frac{1}{2}, B_{0}=C_{0}=F_{0}=G_{0}=\rho_{0}=\delta_{0}=0, E_{0}=\frac{1}{2} \varepsilon=$ const., $A_{1}=0, B_{1}=r, C_{1}=-1, E_{1}=0, F_{1}=-u_{2}+\varepsilon r-\varepsilon u_{2}, G_{1}=-\varepsilon$, $\rho_{1}=-\alpha, \delta_{1}=-\beta, A_{2}=r-2 \alpha \beta, B_{2}=-r_{x}+q r, C_{2}=-q$, $E_{2}=-u_{2}-\varepsilon u_{2}+\varepsilon r+2 \alpha \beta, F_{2}=u_{2 x}+\varepsilon u_{2 x}-\varepsilon r_{x}+2 r u_{1}$ $+\varepsilon q r-q u_{2}-\varepsilon q u_{2}-2 u_{1} u_{2}-2 \varepsilon u_{1} u_{2}+2 \varepsilon r u_{1}, G_{2}=-2 u_{1}$ $-2 \varepsilon u_{1}-\varepsilon q, \rho_{2}=2 \alpha_{x}-q \alpha, \delta_{2}=-2 \beta_{x}-q \beta, A_{3}=-r_{x}+$ $2 q r+4 \alpha_{x} \beta-4 \alpha \beta_{x}-4 q \alpha \beta, B_{3}=r_{x x}-q_{x} r-2 q r_{x}+2 r^{2}+$ $q^{2} r-4 r \alpha \beta-4 \alpha \alpha_{x}, C_{3}=-q_{x}-2 r-q^{2}+4 \alpha \beta+4 \beta \beta_{x}$, $E_{3}=-2 q u_{2}-2 \varepsilon q u_{2}+2 \varepsilon q r+4 r u_{1}+4 \varepsilon r u_{1}-\varepsilon r_{x}-4 u_{1} u_{2}$ $-4 \varepsilon u_{1} u_{2}+u_{2 x}+\varepsilon u_{2 x}-4 \alpha_{x} \beta+4 \alpha \beta_{x}+4 q \alpha \beta, F_{3}=(\varepsilon+1)$. $\left(4 r u_{1}^{2}-4 u_{1}^{2} u_{2}+q_{x} u_{2}+2 q u_{2 x}+2 u_{1 x} u_{2}+4 u_{1} u_{2 x}-4 r_{x} u_{1}-\right.$ $\left.2 r u_{1 x}+2 u_{2}^{2}-u_{2 x x}-q^{2} u_{2}+4 q r u_{1}-4 q u_{1} u_{2}-4 r u_{2}\right)+4 r \alpha \beta$ $+4 \alpha \alpha_{x}+\varepsilon q^{2} r-2 \varepsilon q r_{x}-\varepsilon q_{x} r+2 \varepsilon r^{2}+\varepsilon r_{x x}, G_{3}=-2 u_{1 x}-$ $2 \varepsilon u_{1 x}-\varepsilon q_{x}+2 u_{2}+2 \varepsilon u_{2}-2 \varepsilon r-4 \alpha \beta-4 q u_{1}-4 \varepsilon q u_{1}-\varepsilon q^{2}$ $-4 u_{1}^{2}-4 \varepsilon u_{1}^{2}-4 \beta \beta_{x}, \rho_{3}=-4 \alpha_{x x}+2 q_{x} \alpha+4 q \alpha_{x}-2 r \alpha+2 r_{x} \beta$ $-q^{2} \alpha+4 r \beta_{x}, \delta_{3}=-4 \beta_{x x}-2 q_{x} \beta-4 q \beta_{x}-2 r \beta-4 \alpha_{x}-q^{2} \beta$.

Equations (11) can be written as

$$
\left(\begin{array}{c}
2 A_{m+1}+E_{m+1} \\
-2 C_{m+1}-G_{m+1} \\
2 A_{m+1}+2 E_{m+1} \\
C_{m+1}+G_{m+1} \\
2 \delta_{m+1} \\
-2 \rho_{m+1}
\end{array}\right)=L\left(\begin{array}{c}
2 A_{m+1}+E_{m+1} \\
-2 C_{m}-G_{m} \\
2 A_{m}+2 E_{m} \\
C_{m}+G_{m} \\
2 \delta_{m} \\
-2 \rho_{m}
\end{array}\right),
$$

where

$$
L=\left(\begin{array}{cccccc}
-\partial+\partial^{-1} q \partial & r+\partial^{-1} r \partial & -\partial^{-1} u_{1} \partial & u_{2}+\partial^{-1} u_{2} \partial & \frac{1}{2} \alpha+\partial^{-1} \alpha \partial & -\frac{1}{2} \beta+\partial^{-1} \beta \partial \\
2 & \partial+q & 0 & -2 u_{1} & \beta & 0 \\
0 & 0 & L_{33} & L_{34} & 0 & 0 \\
0 & 0 & -1 & L_{44} & 0 & 0 \\
-4 \beta & -4 \alpha & 2 \beta & -4 \alpha & 2 \partial+q & 2 \\
4 \alpha-4 \beta \partial & 4 r \beta & -2 \alpha+2 \beta \partial & 4 r \beta & 2 r-2 \alpha \beta & -2 \partial+q
\end{array}\right),
$$

$L_{33}=-\partial+\partial^{-1} q \partial+2 \partial^{-1} u_{1} \partial, L_{34}=-2 r+2 u_{2}-2 \partial^{-1} r \partial+2 \partial^{-1} u_{2} \partial$, $L_{44}=\partial+2 u_{1}+q$.

Then, let us consider the spectral problem (7) with the following auxiliary probelm 


$$
\phi_{t_{n}}=V^{(n)} \phi
$$

with

$$
V^{(n)}=\sum_{j=0}^{n}\left(\begin{array}{ccccc}
A_{j} & B_{j} & E_{j} & F_{j} & \rho_{j} \\
C_{j} & -A_{j} & G_{j} & -E_{j} & \delta_{j} \\
0 & 0 & A_{j}+E_{j} & B_{j}+F_{j} & 0 \\
0 & 0 & C_{j}+G_{j} & -A_{j}-E_{j} & 0 \\
\delta_{j} & -\rho_{j} & -\delta_{j} & \rho_{j} & 0
\end{array}\right) \lambda^{n-j}+\frac{1}{2} C_{n+1} e_{1}(0)+\frac{1}{2} G_{n+1} e_{4}(0),
$$

From the compatible condition $\phi_{x, t_{n}}=\phi_{t_{n}, x}$, according to (7) and (14), we get the zero equation

$$
U_{t_{n}}-V_{x}^{(n)}+\left[U, V^{(n)}\right]=0
$$

which gives a nonlinear Lax super integrable hierarchy

$$
u_{t_{n}}=\left(\begin{array}{c}
q \\
r \\
u_{1} \\
u_{2} \\
\alpha \\
\beta
\end{array}\right)_{t_{n}}\left(\begin{array}{c}
C_{n+1, x} \\
B_{n+1}+r C_{n+1} \\
\frac{1}{2} G_{n+1, x} \\
u_{2} C_{n+1}-F_{n+1}-r G_{n+1}+u_{2} G_{n+1} \\
\frac{1}{2} \alpha C_{n+1}-\frac{1}{2} \rho_{n+1} \\
-\frac{1}{2} \beta C_{n+1}+\frac{1}{2} \delta_{n+1}
\end{array}\right)
$$

The super integrable hierarchy (17) is a nonlinear super integrable couplings for the super C-KdV hierarchy

$$
\tilde{u}_{t_{n}}=\left(\begin{array}{c}
q \\
r \\
\alpha \\
\beta
\end{array}\right)_{t_{n}}=\left(\begin{array}{c}
C_{n+1, x} \\
B_{n+1}+r C_{n+1} \\
\frac{1}{2} \alpha C_{n+1}-\frac{1}{2} \rho_{n+1} \\
-\frac{1}{2} \beta C_{n+1}+\frac{1}{2} \delta_{n+1}
\end{array}\right)
$$

\section{Super Hamiltonian STRUCTURES}

A direct calculation reads

$$
\begin{aligned}
& \operatorname{Str}\left(U_{\lambda}, V\right)=-2 A-E, \operatorname{Str}\left(U_{q}, V\right)=2 A+E, \operatorname{Str}\left(U_{r}, V\right)=-2 C-G, \\
& \operatorname{Str}\left(U_{u_{1}}, V\right)=2 A+2 E, \operatorname{Str}\left(U_{u_{2}}, V\right)=C+G, \operatorname{Str}\left(U_{\alpha}, V\right)=2 \delta, \\
& \operatorname{Str}\left(U_{\beta}, V\right)=-2 \rho .
\end{aligned}
$$

Substituting above results into the super trace identity[6,7]

$$
\frac{\delta}{\delta u} \int \operatorname{Str}\left(\frac{\delta U}{\delta \lambda} V\right) \mathrm{d} x=\lambda^{-\gamma} \frac{\partial}{\partial \lambda} \lambda^{\gamma} \operatorname{Str}\left(\frac{\delta U}{\delta u} V\right)
$$

yields that

$$
\frac{\delta}{\delta u} \int(-2 A-E) \mathrm{d} x=\lambda^{-\gamma} \frac{\partial}{\partial \lambda} \lambda^{\gamma}\left(\begin{array}{c}
2 A+E \\
-2 C-G \\
2 A+2 E \\
C+G \\
2 \delta \\
-2 \rho
\end{array}\right)
$$

Comparing the coefficients of $\lambda^{-n-1}$ on both side of (21)

$$
\frac{\delta}{\delta u} \int\left(-2 A_{n+1}-E_{n+1}\right) \mathrm{d} x=\lambda^{-\gamma} \frac{\partial}{\partial \lambda} \lambda^{\gamma}\left(\begin{array}{c}
2 A_{n}+E_{n} \\
-2 C_{n}-G_{n} \\
2 A_{n}+2 E_{n} \\
C_{n}+G_{n} \\
2 \delta_{n} \\
-2 \rho_{n}
\end{array}\right), n \geq 0
$$

From the initial values in (11), we obtain $\gamma=0$. Thus we have

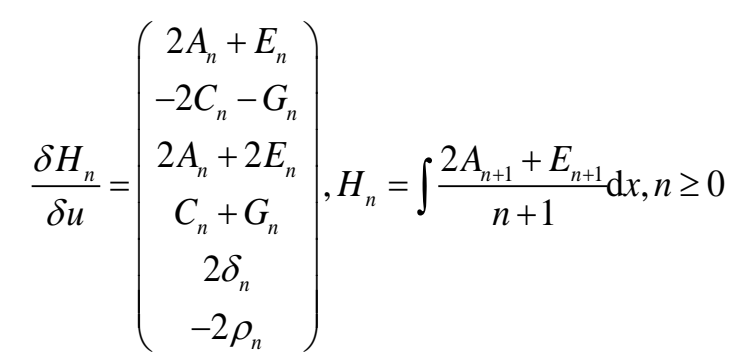

It then follows that the nonlinear super integrable couplings (12) possess the following super Hamiltonian form

$$
u_{t_{n}}=K_{n}(u)=J \frac{\delta H_{n}}{\delta u}
$$

where

$$
J=\left(\begin{array}{cccccc}
0 & -\partial & 0 & -\partial & 0 & 0 \\
-\partial & 0 & \frac{1}{2} \partial & 0 & \frac{1}{2} \alpha & -\frac{1}{2} \beta \\
0 & \frac{1}{2} \partial & 0 & \partial & 0 & 0 \\
-\partial & 0 & \partial & 0 & \frac{1}{2} \alpha & -\frac{1}{2} \beta \\
0 & -\frac{1}{2} \alpha & 0 & -\frac{1}{2} \alpha & 0 & \frac{1}{4} \\
0 & \frac{1}{2} \beta & 0 & \frac{1}{2} \beta & \frac{1}{4} & 0
\end{array}\right)
$$

is a super Hamiltonian operator and $H_{n}(n \geq 0)$ are Hamiltonian functions.

\section{REDUCTIONS}

Taking $\alpha=\beta=0$, the hierarchy (24) reduces to a nonlinear integrable couplings of the $\mathrm{C}-\mathrm{KdV}$ hirerarchy.

When $n=2$ in (24), we obtain the nonlinear super integrable couplings of the second order super C-KdV equatiaons

$$
\left\{\begin{aligned}
q_{t_{2}}= & -q_{x x}-2 r_{x}-2 q q_{x}+4 \alpha_{x} \beta+4 \alpha \beta_{x}+4 \beta \beta_{x x}, \\
r_{t_{2}}= & r_{x x}-2 q_{x} r-2 q r_{x}-4 \alpha \alpha_{x}+4 r \beta \beta_{x}, \\
u_{1, t_{2}}= & (\varepsilon+1)\left(-u_{1 x x}-2 q_{x} u_{1}-2 q u_{1 x}-4 u_{1} u_{1 x}+u_{2 x}\right. \\
& \left.\quad-\frac{1}{2} q_{x x}-r_{x}-q q_{x}\right)+\frac{1}{2} q_{x x}+r_{x}+q q_{x}-2 \alpha_{x} \beta \\
& \quad-2 \alpha \beta_{x}-2 \beta \beta_{x x}, \\
u_{2, t_{2}}= & (\varepsilon+1)\left(-2 q_{x} u_{2}+4 r u_{1 x}-4 u_{1 x} u_{2}-4 u_{1} u_{2 x}-\right. \\
& \left.2 q u_{2 x}+4 r_{x} u_{1}+u_{2 x x}+2 q r_{x}+2 q_{x} r-r_{x x}\right)+ \\
& r_{x x}-2 q_{x} r-2 q r_{x}-4 \alpha \alpha_{x}+4 r \beta \beta_{x}, \\
\alpha_{t_{2}}= & 2 \alpha_{x x}-\frac{3}{2} q_{x} \alpha-2 q \alpha_{x}-r_{x} \beta-2 r \beta_{x}+2 \alpha \beta \beta_{x}, \\
\beta_{t_{2}=}= & -2 \beta_{x x}-2 \alpha_{x}-\frac{1}{2} q_{x} \beta-2 q \beta_{x} .
\end{aligned}\right.
$$


Especially, taking $\alpha=\beta=0$ in (26), we can obtain the nonlinear integrable couplings of the second order $\mathrm{C}-\mathrm{KdV}$ equation

$$
\left\{\begin{aligned}
q_{t_{2}}= & -q_{x x}-2 r_{x}-2 q q_{x}, \\
r_{t_{2}}= & r_{x x}-2 q_{x} r-2 q r_{x}, \\
u_{1, t_{2}}= & (\varepsilon+1)\left(-u_{1 x x}-2 q_{x} u_{1}-2 q u_{1 x}-4 u_{1} u_{1 x}+u_{2 x}\right. \\
& \left.\quad-\frac{1}{2} q_{x x}-r_{x}-q q_{x}\right)+\frac{1}{2} q_{x x}+r_{x}+q q_{x}, \\
u_{2, t_{2}}= & (\varepsilon+1)\left(-2 q_{x} u_{2}+4 r u_{1 x}-4 u_{1 x} u_{2}-4 u_{1} u_{2 x}-\right. \\
& \left.2 q u_{2 x}+4 r_{x} u_{1}+u_{2 x x}+2 q r_{x}+2 q_{x} r-r_{x x}\right)+ \\
& r_{x x}-2 q_{x} r-2 q r_{x} .
\end{aligned}\right.
$$

Let $\varepsilon=0$ in (26), we have

$$
\left\{\begin{aligned}
q_{t_{2}}= & -q_{x x}-2 r_{x}-2 q q_{x}+4 \alpha_{x} \beta+4 \alpha \beta_{x}+4 \beta \beta_{x x}, \\
r_{t_{2}}= & r_{x x}-2 q_{x} r-2 q r_{x}-4 \alpha \alpha_{x}+4 r \beta \beta_{x}, \\
u_{1, t_{2}}= & -u_{1 x x}-2 q_{x} u_{1}-2 q u_{1 x}-4 u_{1} u_{1 x}+u_{2 x} \\
& -2 \alpha_{x} \beta-2 \alpha \beta_{x}-2 \beta \beta_{x x}, \\
u_{2, t_{2}}= & -2 q_{x} u_{2}+4 r u_{1 x}-4 u_{1 x} u_{2}-4 u_{1} u_{2 x}- \\
& 2 q u_{2 x}+4 r_{x} u_{1}+u_{2 x x}-4 \alpha \alpha_{x}+4 r \beta \beta_{x}, \\
\alpha_{t_{2}}= & 2 \alpha_{x x}-\frac{3}{2} q_{x} \alpha-2 q \alpha_{x}-r_{x} \beta-2 r \beta_{x}+2 \alpha \beta \beta_{x}, \\
\beta_{t_{2}}= & -2 \beta_{x x}-2 \alpha_{x}-\frac{1}{2} q_{x} \beta-2 q \beta_{x} .
\end{aligned}\right.
$$

If setting $\varepsilon=-1, u_{1}=-\frac{1}{2} q, u_{2}=r$ in (26), we obtain the second order super $\mathrm{C}-\mathrm{KdV}$ equations

$$
\left\{\begin{array}{l}
q_{t_{2}}=-q_{x x}-2 r_{x}-2 q q_{x}+4 \alpha_{x} \beta+4 \alpha \beta_{x}+4 \beta \beta_{x x}, \\
r_{t_{2}}=r_{x x}-2 q_{x} r-2 q r_{x}-4 \alpha \alpha_{x}+4 r \beta \beta_{x}, \\
\alpha_{t_{2}}=2 \alpha_{x x}-\frac{3}{2} q_{x} \alpha-2 q \alpha_{x}-r_{x} \beta-2 r \beta_{x}+2 \alpha \beta \beta_{x}, \\
\beta_{t_{2}}=-2 \beta_{x x}-2 \alpha_{x}-\frac{1}{2} q_{x} \beta-2 q \beta_{x} .
\end{array}\right.
$$

\section{CONCLUSION}

In this paper, we introduced an approach for constructing nonlinear integrable couplings of super integrable hierarchy. Zhang [19] once employed two kinds of explicit Lie algebra $F$ and $G$ to obtain the nonlinear integrable couplings of the GJ hierarchy and Yang hierarchy, respectively. It is easy to see that Lie algebra $F$ given in [19] is isomorphic to the Lie algebra span $\left\{e_{1}, e_{2}, e_{3}, e_{4}, e_{5}, e_{6}\right\}$ in $g l(6,2)$. So we can obtain nonlinear integrable couplings of super GJ and Yang hierarchy easily. The method in this paper can be applied to other super integrable systems for constructing their super integrable couplings.

\section{REFERENCES}

[1] Y. S. Li and L. N. Zhang, "A note on the super AKNS equations," $J$. Phys. A: Math. Gen, vol. 21, no. 7, pp. 1549-1552, Apr. 1988.

[2] M. H. Tu and J. C. Shaw, "Hamiltonian structures of generalized Manin-Radul super-KdV and constrained super KP hierarchies," $J$. Math. Phys., vol. 40, no. 6, pp. 3021-3034, Jun. 1999.

[3] S. X. Tao and T. C. Xia, "Lie algebra and Lie super algebra for integrable couplings of C-KdV hierarchy," Chin. Phys. Lett., vol. 27, no. 4, pp. 040202, Apr. 2010.

[4] F. J. Yu, "Conservation Laws and Self-Consistent Sources for a Super-Classical-Boussinesq Hierarchy," Chin. Phys. Lett., vol. 28, no. 12, pp. 120201, Dec. 2011.

[5] H. Aratyn, E. Nissimov, and S. Pacheva, "Supersymmetric Kadomtsev -Petviashvili hierarchy: "Ghost" symmetry structure, reductions, and Darboux-Bäcklund solutions," J. Math. Phys., vol. 40, no. 6, pp. 2922-2932, Jun. 1999.

[6] X. B. Hu, "An approach to generate superextensions of integrable systems," J. Phys. A: Math. Gen., vol. 30, no. 2, pp. 619-632, Jan. 1997.

[7] W. X. Ma, J. S. He, and Z. Y. Qin, "A supertrace identity and its applications to super integrable systems," J. Math. Phys., vol. 49, no. 3, pp. 033511, Mar. 2008.

[8] J. S. He, J. Yu, R. G. Zhou, and Y. Cheng, "Binary nonlinearization of the super AKNS system," Mod. Phys. Lett. B, vol. 22, no. 4, pp. 275-288, Feb. 2008

[9] Q. P. Liu, Z. Popowicz, and K. Tian, "Supersymmetric reciprocal transformation and its applications," J. Math. Phys., vol. 51, no. 9, pp. 093511, Sep. 2010.

[10] W. X. Ma, "Enlarging spectral problems to construct integrable couplings of soliton equations," Phys. Lett. A, vol. 316, no. 1-2, pp. 72-76, Sep. 2003.

[11] W. X. Ma, X. X. Xu, and Y. F. Zhang, "Semidirect sums of Lie algebras and discrete integrable couplings," J. Math. Phys., vol. 47, no. 5, pp. 053501, May 2006.

[12] F. K. Guo and Y. F. Zhang, "The quadratic-form identity for constructing the Hamiltonian structure of integrable systems," J. Phys. A: Math. Gen., vol. 38, no. 40, pp. 8537-8548, Oct. 2005.

[13] W. X. Ma and M. Chen, "Hamiltonian and quasi-Hamiltonian structures associated with semi-direct sums of Lie algebras," J. Phys. A: Math. Gen., vol. 39, no. 34, pp. 10787-10801, Aug. 2006.

[14] T. C. Xia and F. C. You, "Multi-component Dirac equations hierarchy and its multi-component integrable couplings system," Chin. Phys. vol. 16, no. 3, pp. 605-610, Mar. 2007.

[15] Y. F. Zhang and E. G. Fan, "Coupling integrable couplings and bi-Hamiltonian structure associated with the Boiti-Pempinelli-Tu hierarchy," J. Math. Phys., vol. 51, no. 8, pp. 083506, Aug. 2010.

[16] Y. F. Zhang and H. Q. Zhang, "A direct method for integrable couplings of TD hierarchy," J. Math. Phys., vol. 43, no. 1, pp. 466-472, Jan. 2002.

[17] W. X. Ma, "Nonlinear continuous integrable Hamiltonian couplings," Appl. Math. Comput., vol. 217, no. 17, pp. 7238-7244, May 2011.

[18] W. X Ma and Z. N. Zhu, "Constructing nonlinear discrete integrable Hamiltonian couplings," Comput. Math. Appl., vol. 60, no. 9, pp. 2601-2608, Nov. 2010.

[19] Y. F. Zhang, "Lie Algebras for Constructing Nonlinear Integrable Couplings," Commun. Theor. Phys., vol. 56, no. 5, pp. 805-812, Nov. 2011.

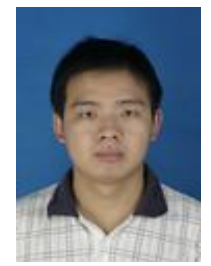

Si-Xing Tao was born in March 1981, in Qingzhou, Shandong Province of China. He received the bachelor degree in Mathematics and Applied Mathematics from Qufu Normal University in 2003 and the master degree in Basic Mathematics from Qufu Normal University in 2006. He received the doctor degree in Basic Mathematics from Shanghai University in 2011. Now he is a lecturer in School of Mathematics and Information Science, Shangqiu Normal University. His research interests are in soliton theory and integrable systems and published more than 10 articles in recent years. 